\title{
Discursos de un grupo de docentes sobre el abordaje del eje de investigación en Lengua y \\ Literatura
}

Discourses of a group of teachers on the approach of the investigation axis in Language and Literature

\author{
Volumen 21, Número 1 \\ Enero - Abril \\ pp. 1-30
}

Carolina Valenzuela Baeza

\section{Citar este documento según modelo APA}

Valenzuela Baeza, Carolina. (2021). Discursos de un grupo de docentes sobre el abordaje del eje de investigación en Lengua y Literatura. Revista Actualidades Investigativas en Educación, 21(1), 1-30. Doi. 10.15517/aie.v21i1.42492 


\title{
Discursos de un grupo de docentes sobre el abordaje del eje de investigación en Lengua y Literatura \\ Discourses of a group of teachers on the approach of the investigation axis in Language and Literature
}

\section{Carolina Valenzuela Baeza ${ }^{1}$}

\begin{abstract}
Resumen: Numerosos estudios han evidenciado los beneficios de desarrollar procesos en los que el alumnado debe investigar. Recientemente, el Marco Curricular nacional, en Chile, ha incluido esta perspectiva como uno de los ejes curriculares junto con los ejes de lectura, escritura y comunicación oral para la asignatura de lengua y literatura. El objetivo de este artículo es determinar en qué medida las prácticas pedagógicas declaradas por un conjunto de docentes favorecen el logro de los objetivos de aprendizaje correspondiente al eje de investigación en lengua y literatura. El artículo se realizó sobre la base del paradigma comprensivo-interpretativo, específicamente, desde una metodología cualitativa y un enfoque basado en la fenomenología hermenéutica. Como técnica de recolección de información, se utilizó la entrevista semiestructurada, la que se aplicó a cinco docentes de lengua y literatura de enseñanza media de la ciudad de Chillán en el año 2019, mientras que el proceso de análisis se llevó a cabo por medio de categorización mixta con el apoyo de Atlas.ti. Los resultados principales indican que el profesorado valora positivamente el eje de investigación, porque contribuye al desarrollo de procesos cognitivos superiores e impacta en el ámbito académico y personal del estudiantado. Sin embargo, lo integran esporádicamente en sus clases porque enfatizan los ejes curriculares contemplados en pruebas estandarizadas. Se concluye que el abordaje del eje de investigación presenta un doble desafío para el profesorado, puesto que le demanda fortalecer y profundizar su propia capacidad crítica y reflexiva para, posteriormente, desarrollar esta capacidad en sus estudiantes.
\end{abstract}

Palabras clave: formación en investigación, práctica pedagógica, educación secundaria, asignatura de lengua y literatura

\begin{abstract}
Numerous studies have shown the benefits of developing processes in which students must investigate. Recently, the national Curricular Framework in Chile has included this perspective as one of the curricular axes along with the axes of reading, writing and oral communication for the subject of Language and Literature. The objective of this article is to determine to what extent the pedagogical practices declared by a group of teachers favor the achievement of the learning objectives corresponding to the axis of research in language and literature. The article was made on the basis of the comprehensive-interpretive paradigm, specifically, from a qualitative methodology and an approach based on hermeneutical phenomenology. As an information gathering technique, the semi-structured interview was used, which was applied to five high school language and literature teachers in the city of Chillán in 2019, while the analysis process was carried out through Mixed categorization supported by Atlas.ti. The main results indicate that the teaching body positively values the research axis, because it contributes to the development of higher cognitive processes and impacts on the academic and personal environment of the student body. However, they integrate it sporadically in their classes because they emphasize the curricular axes contemplated in standardized tests. It is concluded that the approach to the research axis presents a double challenge for teachers, since it requires them to strengthen and deepen their own critical and reflective capacity in order to subsequently develop this capacity in their students.
\end{abstract}

Key words: research training, pedagogical practice, secondary education, language and literature subject

\footnotetext{
${ }^{1}$ Docente y Directora de la Universidad Adventista de Chile, en el Departamento Ciencias de la Educación, Bío Bío, Chile. Magíster en Educación obtenido en la Universidad del Bío-Bío, Bío Bío, Chile. Orcid: https://orcid.org/0000-0001-8626$\underline{3634}$
}

Dirección electrónica: carolinavalenzuela@unach.cl

Artículo recibido: 25 de junio, 2020

Enviado a corrección: 17 de setiembre, 2020 


\section{Introducción}

La educación debe ofrecer al cuerpo estudiantil la posibilidad de desarrollar todas sus capacidades contribuyendo a la formación de personas informadas, responsables, capaces de participar satisfactoriamente en una sociedad democrática enmarcada en el respeto a los derechos de los demás. Para lograr esto es necesario que el estudiantado se enfrente a actividades que impliquen buscar, seleccionar y evaluar información de distintas fuentes haciendo uso del pensamiento crítico y reflexivo. Lo anterior, implica desarrollar la competencia investigativa en el grupo de estudiantes, que no solo les permita acceder al cúmulo de información que hoy en día está disponible en múltiples formatos, sino que también les ayude a avanzar hacia el ejercicio de la autonomía personal y académica. En el proceso educativo la investigación favorece al estudiantado a que puedan organizar sus ideas, conocer sus propias aptitudes para buscar y seleccionar información, ser conscientes de sus errores y puntos débiles, además de conducirlos en la toma de decisiones fundamentadas (Vital, 2015). La investigación, por tanto, debe ser un proceso que se desarrolle en el aula, donde docentes guíen las actividades investigativas, siendo sus estudiantes protagonistas en la construcción de su propio aprendizaje.

Es importante destacar que la Organización para la Cooperación y Desarrollo Económicos (OCDE, 2015) declara que, dentro de las habilidades requeridas para participar activamente en la sociedad actual, se encuentran la alfabetización digital, la creatividad, el pensamiento crítico, la capacidad de resolución de problemas, el trabajo en equipo y la comunicación, es decir, habilidades que implican ir más allá de la simple memorización de hechos y procedimientos. Actualmente, "la sociedad necesita de personas que sean capaces de comprender conceptos complejos, y desarrollar la capacidad para trabajar con ellos de forma creativa y generar nuevas ideas, nuevas teorías, nuevos productos y nuevos conocimientos" (Silva, García, Guzmán y Chaparro, 2016, p.59). En este mismo sentido, se destaca la evidencia disponible que da cuenta de los beneficios que, en sí misma, conlleva la actividad de investigación para el estudiantado, por cuanto se constituye en un factor clave para el proceso de enseñanza y aprendizaje, al transformarse en una herramienta mediadora que permite a docentes, investigadores, estudiantes o aprendices llegar a conocer nuevas ideas y creencias, las que a su vez, entrarán en juego con sus cocimientos previos (Rojas, Naranjo, Campos y Campos, 2018, p. 48). 
EI Ministerio de Educación de Chile (MINEDUC), plantea lo siguiente:

La capacidad para investigar autónomamente permite a un alumno o una alumna guiar su propio estudio, fortalecer su capacidad de juicio y enfrentar de manera informada los desafíos de la vida cotidiana. Por ello, aprender a investigar es una de las metas más importantes de la Educación Media. (Ministerio de Educación del Gobierno de Chile (MINEDUC), 2015, p. 52)

Particularmente en el contexto chileno, durante el año 2015 entraron en vigencia las Bases Curriculares para la enseñanza media o secundaria, y uno de los cambios ocurridos, producto de este nuevo documento curricular, fue que la asignatura Lenguaje y Comunicación sufre reestructuraciones que van desde el cambio de nombre hasta modificaciones más sustanciales, como añadir el componente cultural al enfoque comunicativo ya presente, y cambios a nivel de ejes curriculares.

La nueva asignatura denominada "Lengua y Literatura" dispone objetivos de aprendizaje organizados en los siguientes cuatro ejes curriculares: 1) Lectura 2) Escritura 3) Comunicación Oral e 4) Investigación en Lengua y Literatura. En este último eje, de reciente incorporación, se espera que sus objetivos de aprendizaje permitan al cuerpo estudiantil comunicarse de manera competente, desarrollar un pensamiento crítico y reflexivo frente a ámbitos sociales y culturales, junto con lograr desempeños que les ayuden a discriminar información relevante, determinar la calidad de la información seleccionada y su veracidad para enfrentar los desafíos de la vida diaria. El eje de investigación, por tanto, estaría adoptando un carácter transversal, pues se comprende que "la investigación es un proceso que integra los otros tres ejes de la asignatura y, por lo mismo, es una instancia privilegiada para articular y reforzar los Objetivos de Aprendizaje de Lengua y Literatura" (MINEDUC, 2015, p.52).

La investigación, al constituirse explícitamente en una acción intencionada pedagógicamente desde la estructura curricular vigente, comienza a legitimarse como un quehacer fundamental para el desarrollo de la asignatura, al asumir un rol central como un eje curricular con sus propios objetivos de aprendizaje. De este modo, la investigación deja de ser visualizada solo como una acción de carácter netamente instrumental o utilitaria, para ocupar un espacio propio dentro del currículum. 
Estos cambios, vale decir, nombre y enfoque de la asignatura, incorporación del eje de investigación en lengua y literatura conlleva a analizar las implicancias que tiene, no se trata solo de un cambio superficial, sino que conlleva modificaciones profundas en cómo el profesorado debe comprender la nueva asignatura y, por ende, cómo enseñarla en la sala de clases para realmente alcanzar el propósito planteado.

La investigación científica sobre la enseñanza de la lectura, la escritura y la literatura ha avanzado considerablemente en los últimos años. No obstante, hablar de una didáctica de la lengua y la literatura como una disciplina científica es aún incipiente (Mendoza, 2003; Camps, 2012; Munita y Margallo, 2019); fundamentalmente en lo que respecta al sentido y significado que adopta la acción de investigar dentro de la asignatura de Lengua y Literatura.

A nivel internacional, latinoamericano y chileno, es posible encontrar estudios sobre comprensión lectora, escritura y comunicación oral; entre los que destacan (Iturra, 2015; Silva, 2014; Colomer, 1991) con comprensión lectora; (Espinosa y Concha, 2015; Milián, 2013) sobre escritura, además de (Casanova y Roldán, 2016; Vilà, 2005) con comunicación oral. Sin embargo, el número de investigaciones que abordan la didáctica de la lengua y literatura como una disciplina científica que integra la enseñanza de la lectura, la escritura y literatura de manera articulada es mucho menor.

En relación con estudios que consideren las habilidades investigativas del alumnado, es posible encontrar múltiples investigaciones que se centran en las habilidades investigativas del estudiantado universitario chileno en su proceso formativo. Específicamente, en el ámbito docente, es posible encontrar gran cantidad de estudios latinoamericanos (Soto, 2018; Buendía-Arias, Zambrano-Castillo, y Alirio, 2018; Muñoz, Quintero y Munévar, 2001; Moreno, 2005; Díaz, Solar, Soto, Conejeros y Vergara, 2015) que enfatizan la importancia que tiene el desarrollar las habilidades de investigación en la formación inicial docente, puesto que así el profesorado podrá interpretar, argumentar y proponer alternativas a las posibles problemáticas surgidas en el contexto educativo. Estos estudios señalan que la investigación constituye un desafío en la educación actual, pero también es el camino viable para generar conocimientos, mejorar la labor docente y, por ende, todo el proceso de enseñanza-aprendizaje en las instituciones educativas.

Sin embargo, en la educación secundaria no se evidencia un gran número de estudios que aborden la investigación, ni mucho menos cómo esta se trabaja en la asignatura de lengua y literatura; pareciera ser que la investigación es más trabajada en la educación superior porque dentro del plan de estudios hay asignaturas explícitas que tienen como finalidad brindar 
las herramientas necesarias para que los y las alumnas puedan realizar sus trabajos e investigaciones, y así aportar conocimientos que enriquezcan su disciplina. Dichas investigaciones o trabajos finales de carrera constituyen, por cierto, requisitos de titulación.

Escasa información se encuentra en las revistas especializadas chilenas cuando se indaga en el área de lengua y literatura como una nueva asignatura en el currículum chileno, especialmente en lo relacionado con el eje de investigación presente en la asignatura a partir del año 2015. En lo anterior radica la importancia de este estudio, por cuanto permitirá conocer cómo el profesorado de lengua y literatura está abordando este eje al interior de las aulas secundarias, cómo entienden que debe trabajarse la investigación desde el área de las humanidades, cómo promueven la investigación en su grupo de estudiantes, así como qué significado y valoración le atribuyen al eje.

Lo anterior resulta fundamental debido a la importancia que tiene el saber investigar y acceder a la gran cantidad de información disponible hoy en día. La sociedad actual demanda distinguir las fuentes confiables de las que no lo son; analizar y relacionar lo que están leyendo con los contextos históricos y de producción en el cual fueron creados dichos textos literarios y no literarios; en palabras sencillas, conocer cómo este eje curricular es entendido y trabajado actualmente por docentes, para alcanzar el logro de los objetivos de aprendizaje establecidos en la estructura curricular vigente. Los resultados de este estudio pueden contribuir a optimizar el proceso investigativo en la asignatura de lengua y literatura, a partir de las prácticas pedagógicas declaradas.

\section{Referentes teóricos}

\subsection{Enfoque comunicativo, como base para la asignatura de Lengua y Literatura}

El Ministerio de Educación chileno en su afán de presentar una base curricular común para toda la población estudiantil del territorio nacional, independientemente de su origen, género, condición social y socioeconómica, elaboró las Bases Curriculares de la educación media, que comprende los niveles desde $7^{\circ}$ básico a $2^{\circ}$ medio, abarcando al grupo de estudiantes entre 12 y 16 años. Este documento curricular en la asignatura lengua y literatura, manifiesta explícitamente el propósito de formar estudiantes competentes comunicativamente hablando y que no solo se remitan al aspecto gramatical del lenguaje, guardando estrecha relación con el enfoque comunicativo el cual incorpora un marcado componente cultural (MINEDUC, 2015). 
Este nuevo enfoque conlleva una nueva forma de comprender la enseñanza y el aprendizaje de esta asignatura, pues promueve en el cuerpo estudiantil el desarrollo de capacidades para el uso de la lengua en cualquier situación en la que se puedan encontrar (Mendoza, 2003), pues no basta con que sean comunicativamente competentes solo en la sala de clases, sino que además deben desempeñarse eficazmente en diversos contextos comunicativos. De este modo, se espera que el alumnado asuma un rol activo y protagónico en la construcción de su conocimiento, pues constituiría un apoyo a la hora de elaborar respuestas personales más complejas (Colomer, 2010).

Ruiz (2013) enfatiza en la importancia de saber en qué forma las actividades de enseñanza y aprendizaje que se generan en el aula, permiten a los y las estudiantes construir su conocimiento lingüístico-comunicativo. No obstante, Mendoza (2003) advierte que, en el ámbito escolar, no basta solamente con asegurar el desarrollo de la competencia lingüística, sino que, además, el foco debe estar centrado en asegurar el conocimiento del sistema sociolingüístico, donde el contexto y los escenarios de interacción adoptan relevancia dentro del proceso comunicativo. En palabras sencillas, se privilegia la noción de lenguaje como medio de comunicación situado que tiene lugar en un contexto de interacción específico, no de manera aislada, es decir, ya no se trata solo de hablar correctamente, sino de comunicarse eficiente y efectivamente en diversos contextos o escenarios, tanto en el ámbito académico como personal.

Bérard (como se citó en Beghadid, 2013), señala que el enfoque comunicativo debe considerar las necesidades expresivas del alumnado y trabajar con documentos auténticos de la vida cotidiana como estrategia central para favorecer una mejor y más rápida adquisición de la competencia comunicativa independientemente del contexto. De hecho, la investigación en lengua y literatura no se limita al uso de fuentes bibliográficas, sino que también promueve el recurrir a personas que tengan conocimientos especializados sobre la materia que se investiga (MINEDUC, 2015).

Zebadúa y García (2012) declaran que todo docente de cualquier materia, por el simple hecho de enseñar, se transforma en docente de la lengua que debe fomentar en el estudiantado el diálogo y el respeto por las opiniones diversas. Precisamente la asignatura de lengua y literatura, y en particular el eje de investigación, asume un rol crucial en el currículum chileno, puesto que contribuye a la formación lingüística y literaria del alumnado, al promover actividades de indagación que implican el desarrollo de diversas habilidades como analizar, 
argumentar, reflexionar, discutir, sintetizar, interpretar, entre otras; las cuales van más allá del mero acceso a contenidos curriculares.

La investigación se visualiza como una herramienta que permite al alumnado fortalecer juicios y enfrentar una serie de desafíos que emergen tanto en su vida personal como en su futuro desempeño académico y laboral; además de constituir una forma de aproximación al conocimiento vigente. Por lo tanto, el abordar y desarrollar la investigación al interior del aula, se revelaría como un instrumento de aprendizaje enormemente útil no sólo para la asignatura como tal, sino que para las otras asignaturas del plan de estudio. Vital (2015) considera a la investigación como una estrategia y una herramienta didáctica que genera el desarrollo y habilidades del estudiantado, al mismo tiempo que fortalece el proceso de aprendizaje.

\subsection{Ejes que estructuran la asignatura de Lengua y Literatura}

La asignatura Lengua y literatura está estructurada sobre la base de cuatro ejes curriculares siendo estos lectura, escritura, comunicación oral e investigación, en donde cada eje presenta sus propios objetivos de aprendizaje, los que deben ser trabajados en todas las aulas a nivel nacional. Estos ejes pretenden asegurar el desarrollo de habilidades esenciales en la etapa escolar, pues por una parte promueven el desarrollo de procesos cognitivos mediante actividades de carácter investigativo, mientras que, por otra parte buscan desarrollar la comprensión lectora, expresión oral y expresión escrita, las que resultan necesarias para alcanzar una competencia comunicativa adecuada al nivel (MINEDUC, 2016).

El eje de lectura, busca potenciar en los estudiantes la capacidad de responder competentemente a cualquier desafío de lectura que se presente en la asignatura, independiente del tipo de texto o el soporte en el que éste se encuentre. La lectura, es concebida como la interpretación que los estudiantes hacen de los textos que leen, en donde deben conjugar elementos textuales, objetivos de la lectura y conocimientos previos para construir el texto (MINEDUC, 2015).

Leer no sólo es decodificar un tipo de documento escrito, sino que implica la movilización de conocimientos que el alumnado ya posee de su entorno y experiencias, en donde deben incorporarlos en las lecturas que realizan para otorgarle sentido y por supuesto, comprender lo que se está leyendo explícitamente, como también las inferencias que el texto entrega. El desarrollo del eje de lectura, por tanto, no sólo contribuiría a la asignatura, sino que a todas las asignaturas del currículo por cuanto permite la construcción de significados. 
El eje de escritura se sustenta bajo la idea de concebir a la escritura como una herramienta comunicativa y social fundamental, por lo que cumple una importante función en el desarrollo cognitivo del alumnado. "Las investigaciones han demostrado que escribir ayuda a desarrollar habilidades superiores del pensamiento como organizar, jerarquizar, analizar, sintetizar, evaluar e interpretar" (MINEDUC, 2016, p.45), puesto que no sólo se busca la reproducción de la información, sino que la capacidad de cuestionar premisas, precisar y aclarar ideas previas. Para Giraldo (2015) la escritura es la actividad intelectual que más avances permite en el proceso de aprendizaje, puesto que está vinculada con la capacidad para desarrollar un pensamiento abstracto, objetivo, lógico y racional necesario en el proceso educativo, además de constituir la principal forma de plasmar, transmitir y preservar el conocimiento y la cultura de un país.

El tercer eje, comunicación oral, hace referencia a la oralidad como principal recurso del ser humano para expresar sus ideas, emociones e interactuar con los miembros de la comunidad, puesto que "el lenguaje no solo manifiesta el pensamiento, sino que lo estructura, y el habla da forma a procesos mentales necesarios para el aprendizaje" (MINEDUC, 2016, p.48), por lo que desarrollar habilidades de comunicación oral es clave para formar estudiantes autónomos y autónomas, capaces de construir, compartir y comunicar el conocimiento en una sociedad democrática (MINEDUC, 2016). Lamentablemente no siempre es así, puesto que, en la sala de clases, "el lenguaje oral es principalmente una manera en que el docente transmite conocimientos unilateralmente, verifica lo que saben los alumnos o comprueba si comprendieron el contenido nuevo" (MINEDUC, 2016, p.48).

El último eje corresponde al de investigación, incorporado solo a partir del año 2015, cuya finalidad se centra en potenciar la investigación como medio para el desarrollo de la asignatura, cuya naturaleza fundamentalmente es de carácter bibliográfica y se sustenta en la lectura analítica e interpretativa de variados tipos de textos, la comparación, el contraste de obras desde variados puntos de vista, la búsqueda de posibles relaciones entre ellas y con los diversos contextos en los que nacen y se leen (MINEDUC, 2016), transformándose de este modo en una actividad de indagación que se articula con los otros tres ejes de la asignatura, puesto que al investigar el alumnado deberá leer y analizar diversas fuentes de comunicación, sintetizar y escribir las ideas más relevantes para posteriormente comunicar sus ideas. 


\subsection{Habilidades investigativas}

La investigación en el aula puede ser abordada desde dos perspectivas: la investigación que realiza el profesorado para dar solución a algún problema detectado en el aula, y la investigación que realiza el cuerpo estudiantil orientados por las y los docentes. Para efectos de este estudio, la atención estará puesta específicamente en la investigación dentro de la asignatura de lengua y literatura, principalmente a cómo declaran el profesorado en su discurso abordar y trabajar el eje en el aula. Es importante recordar que el eje de investigación en la asignatura asume un carácter eminentemente bibliográfico, pero no excluye otros tipos de investigación al interior de la asignatura. Pérez y López (1999) definen a las habilidades investigativas como dominio de acciones mentales y prácticas que permiten la regulación racional de la actividad, con ayuda de los conocimientos y hábitos previos para ir a la búsqueda del problema y a la solución del mismo.

Vital (2015) destaca la importancia de enseñar investigando para que los y las estudiantes no reciban los conocimientos ya elaborados, sino que, en la medida de lo posible, los construyan por sí mismos. Intencionalmente la investigación en el aula, actúa como un auxiliar valioso para el desarrollo de la personalidad, puesto que, al enfrentar al alumnado a nuevas situaciones, aumenta la capacidad de acción y comprensión, lo que evitaría formar personas memorísticas y pasivas. Martínez y Marquéz, 2015; Ruíz, 2010 y Chrobak, 2017, afirman que las habilidades investigativas constituyen efectivamente un eje transversal porque permiten integrar el conocimiento, la autocapacitación permanente y la actualización constante de conocimiento, además de articular los otros ejes de currículum, movilizando elementos de distintas disciplinas y ampliando la visión de mundo del grupo estudiantil, lo que fortalecerá su capacidad reflexiva y crítica.

Conocer, por tanto, cómo el estamento docente de lengua y literatura aborda el eje de investigación, permite detectar las creencias que tienen acerca del eje y la valoración que le atribuyen dentro de su asignatura, ya sea como una herramienta útil o simplemente como una actividad más, apartada de los otros ejes curriculares.

\section{Metodología}

\subsection{Enfoque}

El estudio se realizó sobre la base del paradigma comprensivo-interpretativo, específicamente, desde una metodología cualitativa, por cuanto se buscaba lograr una comprensión de los significados que construyen docentes de Lengua y Literatura, a partir del 
análisis de "casos concretos, en su particularidad temporal y local, y a partir de las expresiones y actividades de las personas en sus contextos locales" (Flick, 2012, p.27), Quecedo y Castaño (2002) refuerzan la idea y la importancia que tiene en la investigación cualitativa el hecho de poder acudir y analizar al contexto mismo lo que se desea investigar, en este caso, las personas participantes del estudio trabajan en establecimientos educacionales de enseñanza media o secundaria, y realizan clases de lengua y literatura a más de un nivel educativo. Se empleó un enfoque basado en la Fenomenología Hermenéutica (Van Manen, 2003), porque el interés principal estuvo centrado en lograr la compresión del significado esencial de los fenómenos, su sentido y la importancia que estos tienen en el propósito del contexto de aplicación (Ayala-Carabajo, 2018). Particularmente en este estudio, se buscaba lograr una aproximación a los significados que el profesorado de Lengua y Literatura construye en torno al eje de investigación en la asignatura, a partir de sus vivencias de aula.

\subsection{Unidades de análisis}

Participaron en el estudio cinco docentes que imparten la asignatura de Lengua y Literatura, en dos establecimientos educacionales particulares subvencionados ${ }^{4}$ ubicados en la ciudad de Chillán, Región de Nuble y que cuentan con una mayor matrícula ( $\mathrm{n}^{\circ}$ de estudiantes), lo que asegura, que las personas participantes del estudio, realicen clases en más de un nivel. El personal docente fue incluido a partir de los siguientes criterios internos a la investigación: poseer título profesional de Profesor de Enseñanza Media en Lengua Castellana y Comunicación, realizar docencia en los niveles de $7^{\circ}$ básico a $2^{\circ}$ año de enseñanza media, tener 5 años de experiencia laboral aproximadamente (novatos), puesto que serán docentes que conocen y dominan las bases curriculares recientes, además de disponer de tiempo y voluntad para participar en el estudio (Tabla 1).

\footnotetext{
${ }^{4}$ Establecimiento particular subvencionado: Corresponden a instituciones educativas (colegios) que cuentan con un sostenedor (privado) y aparte reciben subvención económica de parte del Estado de Chile.
} 
Tabla 1

Características de las personas del estudio, Chillán-Chile, 2019

\begin{tabular}{|c|c|c|c|c|c|c|}
\hline Código & $\begin{array}{c}\text { Universidad } \\
\text { de egreso }\end{array}$ & $\begin{array}{c}\text { Años de } \\
\text { experiencia }\end{array}$ & $\begin{array}{c}\text { Título } \\
\text { profesional }\end{array}$ & $\begin{array}{l}\text { Comuna de } \\
\text { procedencia }\end{array}$ & Género & $\begin{array}{c}\text { Tipo de } \\
\text { establecimiento }\end{array}$ \\
\hline P. 1 & Estatal & 5 & $\begin{array}{l}\text { Profesor de } \\
\text { Lengua } \\
\text { Castellana y } \\
\text { Comunicación }\end{array}$ & Chillán & Femenino & $\begin{array}{l}\text { Particular } \\
\text { subvencionado }\end{array}$ \\
\hline P. 2 & Estatal & 6 & $\begin{array}{l}\text { Profesor de } \\
\text { Lengua } \\
\text { Castellana y } \\
\text { Comunicación }\end{array}$ & Chillán & Masculino & $\begin{array}{l}\text { Particular } \\
\text { subvencionado }\end{array}$ \\
\hline P. 3 & Estatal & 5 & $\begin{array}{l}\text { Profesor de } \\
\text { Lengua } \\
\text { Castellana y } \\
\text { Comunicación }\end{array}$ & Chillán & Masculino & $\begin{array}{l}\text { Particular } \\
\text { subvencionado }\end{array}$ \\
\hline P. 4 & Estatal & 5 & $\begin{array}{l}\text { Profesor de } \\
\text { Lengua } \\
\text { Castellana y } \\
\text { Comunicación }\end{array}$ & Chillán & Femenino & $\begin{array}{l}\text { Particular } \\
\text { subvencionado }\end{array}$ \\
\hline P. 5 & Estatal & 7 & $\begin{array}{l}\text { Profesor } \\
\text { Español }\end{array}$ & Chillán & Femenino & $\begin{array}{l}\text { Particular } \\
\text { Subvencionado }\end{array}$ \\
\hline
\end{tabular}

Fuente: Elaboración propia, 2019

\subsection{Técnica de recolección}

Para obtener la información requerida, se aplicó como técnica de recolección de información la entrevista semiestructurada, entendida como un tipo de herramienta que permite al investigador "encontrar lo que es importante y significativo en la mente de los informantes, sus significados, perspectivas e interpretaciones, el modo en que ellos ven, clasifican y experimentan su propio mundo" (Ruiz, 2012, p. 166). La entrevista fue aplicada a 5 docentes ( 3 mujeres y 2 hombres) de enseñanza media que llevan a cabo sus prácticas en los niveles de $7^{\circ}$ a $2^{\circ}$ medio, de la asignatura lengua y literatura. La entrevista, se organizó bajo un guion de preguntas orientadoras, puesto que permiten a la persona entrevistadora tener la libertad de introducir preguntas adicionales, ya sea para precisar ciertos conceptos o para profundizar en algún punto (Hernández, Fernández y Baptista, 2014), propiciando de esta forma, un diálogo que busca conocer desde la voz del profesorado sus valoraciones y prácticas, respecto del eje de investigación. La entrevista se realizó de la siguiente manera:

1. Elaboración de un guion de preguntas redactadas de forma previa y validada por el juicio de personal experto, vale decir, con trayectoria en el tema y reconocidas por los demás como personas cualificadas en un área determinada, y que pueden dar información, 
evidencia, juicios y valoraciones (Escobar-Pérez y Cuervo-Martínez, 2008). En este caso en particular, corresponden a cinco personas especialistas en educación y en didáctica de la lengua y la literatura. Tras someter en este caso, el guion de preguntas al juicio de personal experto, se procuró resguardar la validez tanto del diseño del instrumento, como del contenido; de manera tal que fuera comprensible para todo el cuerpo docente, para esto, cada miembro del comité experto asignó un puntaje de uno a cinco a cada pregunta, además de dejar su observación en el espacio para comentarios; de esta forma, se realizó una revisión y ajustes a cada ítem del instrumento final. Finalmente, se realizó lo que Robles y Rojas (2015) señalan como la labor fundamental de todo investigador o investigadora, es decir "eliminar aspectos irrelevantes, incorporar los que son imprescindibles y/o modificar aquellos que lo requieran" (p.1). De este modo, se veló por la pertinencia, claridad y coherencia del instrumento, siguiendo las recomendaciones derivadas del juicio del personal experto.

2. Se aplicó la entrevista a cada docente en forma directa, quienes fueron informados sobre la finalidad de la investigación, asegurándoles la confidencialidad de sus datos, además de firmar voluntariamente un consentimiento informado en el que se especificaba por escrito, lo mismo que se conversó con el/la docente.

3. El discurso del profesorado fue grabado en formato audio y posteriormente, transcrito de manera literal.

\subsection{Procesamiento de análisis}

Para el análisis de la información se empleó un proceso de categorización de carácter mixto, es decir, deductivo e inductivo (Tabla 2). Desde el punto de vista deductivo, se definieron apriorísticamente categorías de análisis en función de los objetivos definidos para el estudio, mientras que, desde la perspectiva inductiva, se consideraron códigos que fueron emergiendo en el trabajo de campo, desde el discurso de los participantes. Todo el proceso de análisis fue apoyado por el software Atlas.ti v.8 (Figura 1). 
Tabla 2

Categorías y códigos definidos para el análisis del estudio, Chillán-Chile, 2019

\begin{tabular}{|c|c|c|c|}
\hline Categoría & Subcategoría & Códigos & Descripción \\
\hline \multirow[t]{2}{*}{$\begin{array}{l}\text { Prácticas } \\
\text { docentes } \\
\text { desde las } \\
\text { voces del } \\
\text { profesorado }\end{array}$} & $\begin{array}{l}\text { MDV: Metodología } \\
\text { docente desde la } \\
\text { voz }\end{array}$ & $\begin{array}{l}\text { MDV1.Objetivo de la clase } \\
\text { MDV2. Metodología para abordar el eje } \\
\text { MDV3. Recursos materiales empleados } \\
\text { MDV4.Evaluación del eje } \\
\text { MDV 5.Criterios para la planificación }\end{array}$ & \multirow{2}{*}{$\begin{array}{l}\text { Identificación } \\
\text { de las prácticas } \\
\text { docentes } \\
\text { declaradas de } \\
\text { los profesores } \\
\text { de lengua y } \\
\text { literatura, } \\
\text { orientadas a } \\
\text { favorecer los } \\
\text { objetivos de } \\
\text { aprendizaje del } \\
\text { eje, desde el } \\
\text { discurso }\end{array}$} \\
\hline & $\begin{array}{l}\text { EDV: Expectativa } \\
\text { docente desde la } \\
\text { voz }\end{array}$ & EDV1.Tratamiento del eje & \\
\hline \multirow[t]{3}{*}{$\begin{array}{l}\text { Valoración del } \\
\text { eje de } \\
\text { investigación }\end{array}$} & $\begin{array}{l}\text { EVE: Valoración del } \\
\text { eje }\end{array}$ & $\begin{array}{l}\text { EVE1. Priorización de otros ejes } \\
\text { EVE2. Disponibilidad de recursos } \\
\text { EVE3. Evaluaciones estandarizadas }\end{array}$ & \multirow{3}{*}{$\begin{array}{l}\text { Valoración de } \\
\text { los docentes de } \\
\text { lengua } \\
\text { literatura sobre } \\
\text { el eje de } \\
\text { investigación, a } \\
\text { partir del } \\
\text { discurso del } \\
\text { profesorado }\end{array}$} \\
\hline & $\begin{array}{l}\text { IE: Importancia del } \\
\text { eje }\end{array}$ & IE1: Importancia del eje & \\
\hline & $\begin{array}{l}\text { EDA: Expectativa } \\
\text { docente desde la } \\
\text { acción }\end{array}$ & EDA1.Tratamiento del eje & \\
\hline
\end{tabular}

Fuente: Elaboración propia, 2019

Figura 1

Red de categorías y códigos empleados para el análisis de la información proporcionada por docentes de Lengua y Literatura, Chillán-Chile, 2019

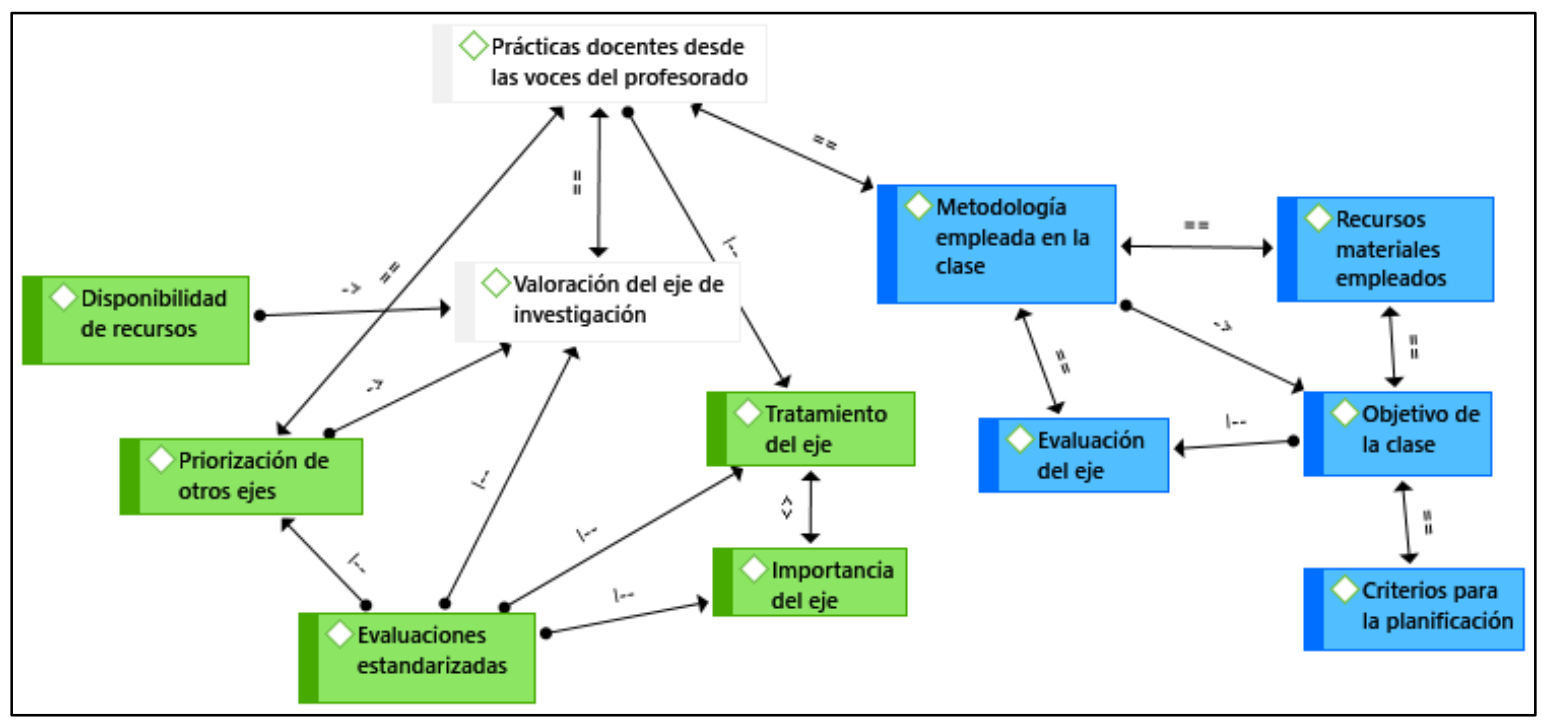

Fuente: Elaboración propia con información de los participantes, 2019. 


\section{Resultados}

El objetivo de este artículo es determinar en qué medida las prácticas pedagógicas declaradas de un conjunto de docentes favorecen el logro de los objetivos de aprendizaje correspondiente al eje de investigación en lengua y literatura, esto, debido a la importancia que tienen las habilidades de investigación para el alumnado, no sólo en el plano académico, sino también en lo personal y en su futura vida laboral.

A partir de los resultados de este estudio, es posible inferir que para el profesorado la acción de investigar es asimilable a una búsqueda de información centrada más bien en la profundización de información, descartando alternativas de investigación bibliográfica que tributen al desarrollo de habilidades tales como seleccionar correctamente la información, comparar diversas fuentes entre sí, relacionar información, para desarrollar procesos cognitivos superiores que favorezcan el aprendizaje, por lo que la investigación, se enmarcaría dentro de una dimensión utilitaria e instrumental, puesto que se utiliza más bien como una actividad de búsqueda de información, más que una estrategia que permita despertar la curiosidad del alumnado, plantearse interrogantes y dar respuesta esas inquietudes.

Es importante señalar, además que el eje de investigación es propio de la asignatura Lengua y Literatura y busca desarrollar competencias comunicativas en los estudiantes, al mismo tiempo que tributa a los otros ejes curriculares de la asignatura, es decir, la lectura, escritura y oralidad.

\subsection{Valoración del eje de investigación, desde la perspectiva del profesorado}

En lo que respecta a la valoración que se le atribuye a este eje, llama la atención que el profesorado participante le atribuye una alta importancia, argumentando que aporta en la vida académica, personal y para el futuro laboral del grupo estudiantil. Frente a ello, señalan que debería ser una actividad trabajada de manera transversal y no sólo en la asignatura de Lengua y Literatura. Esta valoración claramente está en la línea de lo que sostiene el MINEDUC (2016), en tanto la investigación se visualiza como una instancia donde la lectura, escritura y comunicación oral se articulan y tributan al logro de los aprendizajes, así como también en lo que señalan diversos autores, que consideran a la investigación como un eje transversal dentro de la educación (Chrobak, 2017; Martínez y Marquéz, 2015; Ruíz, 2010).

La investigación, además de poner en acción los otros ejes curriculares, permitiría a estudiantado tener una visión más completa de la historia de su país y por ende del patrimonio cultural, lo que ampliará su visión de mundo y fortalecerá su capacidad reflexiva y crítica al 
tener conciencia de su propia cultura (MINEDUC, 2015), por cuanto pueden acceder a conocer distintas épocas y movimientos culturales, más allá del aula, que existieron en el mundo y contraponerlos con la realidad chilena, para así, apreciar distintas visiones de mundo y manifestaciones culturales, como por ejemplo, la música.

A través del discurso del profesorado es posible señalar que la totalidad comprende la investigación en lengua y literatura como una actividad más bien bibliográfica, basada en la búsqueda de información obtenida principalmente a través de internet, puesto que, al usar libros, por lo general los y las estudiantes no prestan la misma atención. El cuerpo docente agrega que es importante trabajar el eje de investigación en la etapa de escolarización en la que se encuentra el alumnado de educación media, aludiendo a que éste no sólo debería abordarse en la asignatura de lengua y literatura, sino que debería ser una habilidad que se promueva en forma transversal, argumentando que para el alumnado el hecho de adquirir y desarrollar la habilidad de investigar les resulta beneficioso para su vida personal, académica y laboral. No obstante, también reconocen que es un eje escasamente intencionado en sus prácticas docentes:

Considero que es súper importante, pero en el trabajo en la realidad no se le da la importancia que debería tener, por lo mismo que mencionaba que se preocupa del currículum, si tú me dices importancia, la verdad no se le da importancia al eje no porque no lo merezca, sino que se les da importancia a otras, al desarrollo de otras habilidades. (Profesora 1, comunicación personal, 30 de agosto, 2019)

La investigación a mi parecer es importante que se potencie desde la etapa escolar, pero debería ser desde todas las asignaturas y no sólo de unas cuantas. (Profesor 2, comunicación personal, 02 de septiembre, 2019)

A ellos les sirve ahora en su etapa escolar para investigar sobre temas académicos, pero también personales, de aspectos de sus vidas y que desean conocer más; pero también les sirve y les servirá en su vida personal, por ejemplo, para investigar de la carrera que les gustaría estudiar, cómo ayudar al planeta, cómo nació un movimiento musical, y una infinidad de otros temas más que pueden encontrar en internet. (Profesora 5, comunicación personal, 08 de octubre, 2019) 
El personal docente participante del estudio valora positivamente la incorporación del eje, pero declaran no trabajarlo en sus aulas ni otorgar los espacios necesarios para hacerlo, es decir el eje se trabaja más bien a nivel declarativo que práctico, el profesorado argumenta no trabajar el eje de investigación, porque prioriza otros ejes curriculares, principalmente al de lectura, por ser considerado en las evaluaciones estandarizadas. Esta situación se presenta de igual forma en un estudio realizado por Díaz et al. (2015) quienes concluyen que tanto profesores y profesoras del sistema escolar como universitario chileno, muestran interés por empoderarse de una cultura innovadora, otorgando un gran valor a investigación; pero al mismo tiempo investigan poco. Dentro de las causas esto últimos docentes declarado tener condiciones labores poco favorables para investigar, como, por ejemplo, la falta de tiempo para dedicarse a la investigación, tanto a nivel universitario como escolar.

\subsubsection{Priorización de otros ejes curriculares}

El profesorado también declara explícitamente que relegan el eje de investigación a un segundo plano, debido a que priorizan los otros ejes del currículum, principalmente los de lectura y escritura, para dar respuesta a exigencias externas impuestas desde el propio Ministerio de Educación, tales como evaluaciones estandarizadas aplicadas de manera obligatoria en el país (SIMCE ${ }^{5}$ y Prueba de Selección Universitaria-PSU). Esta escasa consideración del eje de investigación por parte del profesorado, puede ser explicada a partir de lo expuesto por Manzi, Bogolasky, Gutiérrez, Grau y Volante (2014), cuando advierten que la difusión de los resultados SIMCE se vincula al prestigio del establecimiento educacional, lo que desencadena "distorsión de las prácticas pedagógicas, lo que se daría al centrar la atención y el uso del tiempo en las asignaturas evaluadas por el SIMCE en desmedro de las demás" (p.18); reduciendo de esta forma las áreas que no son evaluadas por la prueba. Del mismo modo, Falabella y Opazo (2014) hacen alusión al agobio que reciben los y las docentes que realizan clases en niveles que rinden SIMCE, dado por la presión que tienen por obtener buenos puntajes; esto conlleva a los docentes a relegar el eje de investigación a un segundo plano.

5 SIMCE: Sistema de Medición de Calidad de la Educación. Es una evaluación de aprendizaje que aborda el logro de los contenidos y habilidades del currículo vigente en diferentes asignaturas y áreas de aprendizaje, y que se aplica a todos los estudiantes del país que cursan los niveles evaluados. En el caso de la asignatura lengua y literatura, las áreas evaluadas son lectura y escritura. 
Las razones que esgrimen para no trabajarlo de forma intencionada en sus clases, se enmarcan dentro de la priorización que realizan de los ejes el de lectura y escritura, en tanto son aquellos que las pruebas estandarizadas, como el SIMCE consideran como foco evaluativo. Del mismo modo, hacen referencia a la carencia de recursos que se observan en los establecimientos educacionales, como ejemplo, no contar con computadores para todo el estudiantado, junto con la falta de tiempo para preparar actividades donde además de abordar el eje, puedan trabajar temáticas actuales que sean del interés del alumnado y se relacione con lo trabajado en la asignatura; generando así mayor motivación. Otro punto importante de destacar, además de realizar las actividades de manera grupal, es que los y las docentes asocian el eje de investigación con el uso de las tecnologías, por sobre textos es soporte impreso.

Tampoco se le atribuye mucha importancia porque se le da prioridad al desarrollo de otras habilidades como la comprensión lectora y la escritura que insisto, uno como profesora cae en ese juego de preparar para el SIMCE, pero en lo personal considero que es importante desarrollar y trabajar este eje. (Profesor 3, comunicación personal, 09 de septiembre, 2019)

La investigación es importante en todas las etapas del sistema escolar. Ahora haciendo un mea culpa, en mi asignatura no trabajamos tanto el eje, porque conlleva mucho tiempo, no es que no queramos hacerlo, pero siempre los énfasis de este colegio y en general en la mayoría, es potenciar habilidades de escritura y comprensión por las famosas pruebas estandarizadas, que, al fin y al cabo, con los resultados obtenidos en esas evaluaciones, son medidos los colegios (Profesor 2, comunicación personal, 02 de septiembre, 2019)

Investigaciones internacionales apoyan lo declarado por el profesorado con respecto a la sobrecarga y exigencias que tienen que cumplir producto de las evaluaciones estandarizadas Dooley y Assaf (2009) señalan que docentes de lectura y escritura en Estados Unidos están sintiendo una gran presión para mejorar los puntajes de las pruebas, sintiendo incluso presiones para limitar sus prácticas de instrucción a la instrucción de habilidades de bajo nivel. Sánchez y del Sagrario Corte (2013) señalan que 
después de un cuarto de siglo de aplicación de exámenes estandarizados nacionales e internacionales, tanto a los alumnos como a los maestros, en diversos países de América Latina, las evidencias empíricas muestran que el aprovechamiento escolar de los alumnos ha disminuido (p.116)

El profesorado también manifiesta no abordar el eje de investigación muchas veces por la escasez de los recursos materiales, más bien del tipo tecnológico, lo que se profundizará más adelante:

Yo creo que a veces los profesores de lenguaje, dejamos un poquitito de lado el tema de investigación, puede ser a lo mejor porque muchas veces en los colegios no tenemos como los espacios, o por ejemplo el tema de llevar el laboratorio móvil a la sala, no nos sirve mucho por el tema del internet, entonces lo dejamos como un poquito de lado (Profesora 4, comunicación personal, 05 de octubre, 2019)

A raíz de lo declarado por el cuerpo docente se puede observar una tensión existente entre el discurso y lo práctico, que a su vez responde a mandatos externos, como son las exigencias y sobrevaloración de las evaluaciones estandarizadas. Junto con esto, resulta importante señalar que para las personas participantes de este estudio pareciera ser que la lectura es vista como una habilidad aparte o desligada de la investigación, por cuanto señalan privilegiar el eje de lectura por sobre el eje de investigación.

\subsection{Metodología implementada en la clase para favorecer el eje de investigación, desde las voces del profesorado}

Desde el punto de vista curricular, fundamentalmente en lo referido a cómo planifican sus clases cuando deciden considerar el eje de investigación, el profesorado declara que los objetivos de aprendizaje presentes en los programas de estudio elaborados por el Ministerio de Educación por una parte son ambiguos, porque en el propósito de las asignatura se declaran como objetivos muy ambiciones, pero los tipos de actividades sugeridos en los programa de estudio, siguen siendo las mismas de siempre; y por otra son muy extensos, por lo que los y las docentes deben optar por desglosarlos y abordarlos en más de una clase. En este mismo sentido, señalan que cuando seleccionan textos y/o actividades desde el texto escolar, dentro de los aspectos que consideran para dicha selección se encuentra las 
características del contexto y la realidad del alumnado, destacando que escogen textos con temáticas actuales, pero que no sean extensos,

Ya, primero hay que revisar el objetivo, la duración de la clase, por ejemplo, hay textos que vienen en el libro, el texto del estudiante, que no siempre sirven por lo mismo, porque la extensión no es la adecuada, que sea de interés, ojalá de temas contingentes o relacionado a la unidad que se está trabajando en el momento. (Profesor 3, comunicación personal, 09 de septiembre, 2019)

Lo principal para mí a la hora de planificar, es considerar la realidad de los estudiantes, porque sólo así puedo lograr que para ellos sea importante lo que están aprendiendo, y junto con esto, busco que lo que enseño, sean más bien habilidades porque eso nos pide el SIMCE y la vida después de egresar del colegio (Profesora 1, comunicación personal, 30 de agosto, 2019)

El eje de investigación y el objetivo que nos plantea el programa es súper amplio, entonces abarcarlo en una clase completa no se puede, por qué, por la realidad de estudiantes que tenemos hoy en día, entonces qué se hace, se desglosa el objetivo de aprendizaje y se divide en diferentes clases, o bien tomo solamente una parte del objetivo de aprendizaje (Profesora 4, comunicación personal, 08 de octubre 2019)

Lo señalado por la profesora es totalmente coherente con la forma de trabajar los objetivos de aprendizaje, de hecho, se busca que los objetivos sean macros e involucren a los distintos ejes curriculares y sean los profesores quienes, desde su experticia y contexto educativo, puedan ir desglosando para trabajar clase a clase con metas más reales y concretas.

En relación a la forma en cómo dicen abordar el eje de investigación en sus clases cuando lo hacen- se puede evidenciar un predominio de estrategias vinculadas a la organización estudiantil en grupos y al empleo de recursos TIC

La estrategia principal que me resulta es la de trabajar en grupos, pero siempre y cuando sean por afinidad. (Profesor 2, comunicación personal, 02 de septiembre, 2019)

Cuando decido trabajar el eje de investigación, los estudiantes buscan información en internet y luego la escriben en su cuaderno o bien, los hago exponer frente a sus compañeros [...]. Es súper difícil abordar ese eje... ¡ hay que ser súper ingenioso!, porque 
a veces uno lo aborda solamente desde las Tic's y hay centros en los que no están las condiciones necesarias para trabajarlo a través de ese medio, por lo tanto, hay que ingeniárselas, llevar o dar material. (Profesora 5, comunicación personal, 08 de octubre, 2019)

Al ser consultados los docentes acerca de qué metodologías son las que más utilizan a la hora de abordar el eje de investigación, el profesorado declara privilegiar el trabajo en grupo, hacer uso de recursos tecnológicos y sobretodo de la creatividad del docente para cautivar a sus estudiantes. Básicamente las estrategias que utilizan para trabajar la investigación en lengua y literatura es buscar información adicional a la clase o que pueda enriquecer a la clase, en otras palabras, la investigación en lengua y literatura, se entiende más como sinónimo de buscar información. Dentro de las respuestas que entregó el personal docente, no se hizo alusión por ejemplo a cómo enseñan a discriminar fuentes confiables de las que no son, es el docente quien les dice en qué buscador deben realizar sus investigaciones, tampoco se señala qué, deben hacer con la información nueva ni cómo deben incorporarla en los trabajos escritos. Básicamente en el eje de investigación el estudiantado asume un rol bastante pasivo, puesto que tienen que buscar información para contestar preguntas realizadas previamente el cuerpo docente. Resultaría interesante poder conocer desde la acción docente cómo los y las docentes retroalimentan las investigaciones realizadas por sus estudiantes y el uso que hacen de ella en la sala de clases.

\subsubsection{Recursos materiales empleados y evaluación del eje de investigación}

Dentro de los recursos que el profesorado señala emplear, se evidencia el predominio de textos digitales, porque, al decir de ellos, de esta forma logran motivar mayormente al cuerpo estudiantil. También se evidencia un mayor énfasis en el uso de textos no literarios, principalmente, expositivos e informativos, aludiendo a que este tipo de texto implica un menor esfuerzo para sus estudiantes

Cuando trabajamos la investigación, les pido a los estudiantes que usen videos, textos digitales, principalmente noticias de actualidad y biografías de autores para enriquecer las lecturas que ellos hacen. $Y$ decido hacerlo de esta forma, porque si les pido investigar de otra forma, por ejemplo, en la biblioteca no lo hacen. (Profesora 1, comunicación personal, 30 de agosto, 2019) 
Eran textos no literarios, textos informativos, porque los textos literarios la verdad es que no he llegado a una actividad o no he creado a una actividad en donde puedan investigar sobre algo, un personaje por ejemplo de cuento, de novela, etc., generalmente se trabaja con los textos que son expositivos e informativos, porque es más fácil trabajar con ellos. (Profesor 3, comunicación personal, 09 de septiembre, 2019)

Cuando abordo el tema de investigación, textos físicos no, generalmente recurro a páginas de internet. (Profesor 2, comunicación personal, 02 de septiembre, 2019)

Es importante destacar este punto respecto a los tipos de textos utilizados para abordar la investigación, pues deja en evidencia la comodidad docente al trabajar con textos que son más bien sencillos, por sobre textos que implicarían mayores desafíos para el alumnado. Sumado a lo anterior, se evidencia una subvaloración de la actividad investigativa que lleva a cabo el estudiantado cuando se les solicita algún tipo de trabajo de indagación, aludiendo a que no tienen mayor conocimiento sobre la calidad y fiabilidad de las páginas web que visitan y/o desde dónde extraen la información, por lo que también es necesario dedicar tiempo a alfabetizarlos informacionalmente:

Yo creo que los chicos de hoy en día creen que saben investigar, pero en realidad no saben. No es tan simple como ellos creen porque toda la información que tienen que seleccionar a lo mejor no se va a dar con tanta facilidad en las páginas que siempre buscan, páginas que no son fidedignas ni confiables como lo son Wikipedia, el Rincón del Vago, el Vago escolar, Yahoo respuestas, entre otras muchas. Por ejemplo, nosotros con la educadora diferencial, hemos tenido que abrir Google en la sala de clases y enseñarles a utilizar el Google académico y ellos ni sabían que existía. (Profesora 4, comunicación personal, 08 de octubre, 2019)

Les falta mucho desarrollar las habilidades investigativas porque las investigaciones que ellos hacen son bien sencillas, y no seleccionan fuentes de información confiables, seleccionan lo primero que encuentran. (Profesora 1, comunicación personal, 30 de agosto, 2019) 
Lo anterior es relevante, puesto que, bajo esta lógica, el profesorado reconoce que el alumnado realiza investigaciones incipientes y que resultan realmente desafiantes, porque el estudiantado no contaría con las habilidades para desarrollar las investigaciones que se les solicitan. Sin embargo, manifiestan no trabajar intencionadamente el eje de investigación en sus aulas, aun cuando saben que constituye una debilidad en sus estudiantes, puesto que privilegian otros ejes curriculares. De esto se desprende el doble desafío del profesorado: pensar críticamente y promover el pensamiento crítico, pues "la realidad es que los maestros serán capaces de fomentar el pensamiento crítico solo en la medida en que ellos mismos piensen críticamente". (Paul y Elder, 2005, p.6).

De lo anterior es posible referirse a las expectativas que tiene el profesorado respectos del alumnado y sus habilidades para investigar, por una parte, declaran utilizar mayoritariamente textos expositivos e informativos porque son más fáciles de trabajar, lo que haría suponer que un texto de mayor dificultad representaría realmente un mayor desafío. Por otro lado, asumen que el cuerpo estudiantil debería conocer y dominar buscadores y páginas más bien académicas, puesto que se sorprenden que no sepan cómo buscar información. Producto de esto, es que una de las personas entrevistadas tuvo que destinar una clase para enseñarlo. Se despende de lo anterior, el rol que como profesores y profesoras debemos tener en la sala de clases, no se trata sólo de mostrar cómo deben, en este caso, buscar información, sino ser capaces de guiar al estudiantado para que sean ellos quienes busquen, revisen y escojan finalmente las fuentes que permitan sustentar sus indagaciones. Si como docente se asume que el estudiantado sabe investigar, claramente podría encontrarse con realidades muy variadas. Torres (1999) indica que la investigación en el aula promovida por los y las docentes, propicia en el alumnado la curiosidad, la necesidad de saber, de preguntar, de explorar, de comprobar, de experimentar, de perfeccionar, estimulando en ellos el deseo de aprender y no el aprender por miedo ni por obligación. Por este motivo, el rol y las acciones del profesorado son clave para el desarrollo de las habilidades de investigación, el pensamiento creativo y crítico.

Finalmente, en lo referido a las estrategias evaluativas que implementan para valorar los objetivos de aprendizaje asociados al eje de investigación, los y las docentes señalan que utilizan varios instrumentos, tales como pautas y rúbricas, y dentro de los tipos de evaluación, de preferencia la autoevaluación: 
Ahí se aplican distintas formas de evaluación y la más importante, y con la que me quedo es con la autoevaluación, la idea era que ellos no se evaluaran el producto, sino que el proceso, que fueran capaces de identificar las dificultades o aquello donde se le había dado más fácil el trabajo. (Profesor 3, comunicación personal, 08 de octubre, 2019) Con rúbricas, pautas y a veces alguna autoevaluación. (Profesor 2, comunicación personal, 02 de septiembre, 2019)

Yo creo que los mecanismos que se permiten evaluar el eje, son el trabajo en equipo...el hecho saber buscar en las fuentes pertinentes respectos a un tema, y el hecho de trabajar algo que no sea tradicional, porque eso igual motiva a los alumnos de una manera distinta, porque si hago después una prueba de la investigación eso lo toman de una manera distinta, se produce un rechazo. (Profesora 1, comunicación personal, 30 de agosto, 2019)

Finalmente, la escasa consideración del eje de investigación por parte del profesorado a nivel de sus prácticas pedagógicas declaradas también puede ser discutido a partir del histórico proceso de desprofesionalización de la labor que lleva a cabo el profesorado, pues desde la política pública vinculada a educación, el docente ha ido asumiendo un rol más bien de carácter técnico, en tanto se ha visto en la necesidad de implementar sus prácticas pedagógicas a partir de lo que otras personas (expertas) le dicen qué hacer y cómo hacerlo, por ello se concuerda con Viñao (2018), cuando señala que:

El profesorado no es hoy quien, desde su oficio, elabora el saber científico-pedagógico. A lo sumo, se limita a recibirlo y reinterpretarlo, en función del contexto, cuando lo considera pertinente y puede. Es lógico que sea así: la mayor parte de dicho saber se hace desde intereses y criterios al margen del oficio. (p.10)

Sumado a ello, aparece el componente asociado a la autonomía profesional, porque si bien desde el currículum nacional se define el qué se debe enseñar por medio de los objetivos de aprendizaje, el profesorado tiene libertad al interior del aula para contextualizar el currículum, para seleccionar y utilizar variadas herramientas didácticas que favorezcan aprendizajes de calidad en el alumnado, pero dicha autonomía y dicho proceso deliberativo no se ejerce en plenitud, principalmente por el temor que les generan las evaluaciones estandarizadas, o bien, por el esfuerzo que implica la búsqueda de alternativas metodológicas 
y didácticas variadas para favorecer el aprendizaje, sumado esto, hay que recordar que el eje de investigación en lengua y literatura fue recientemente incorporado en el currículum y no es foco evaluativo dentro de las pruebas obligatorias.

\section{Conclusiones}

El reconocimiento de la importancia que tiene la investigación en materia educativa, y específicamente cómo se aborda la investigación en el área de las humanidades, haría suponer el desarrollo de múltiples investigaciones en torno a lo que significa trabajar y promover la investigación al interior del aula según lo planteado en las Bases Curriculares, con estudios que por una parte describieran y analizaran las prácticas docentes en la enseñanza de la nueva asignatura Lengua y Literatura, con énfasis en el eje de investigación, y por otra; entregaran sugerencias $u$ orientaciones claras y puntuales para la formación inicial y continua de profesores y profesoras que deben abordar y enseñar el eje. Debido a lo anterior, este artículo, tuvo como propósito determinar en qué medida las prácticas pedagógicas declaradas de un conjunto de profesores y profesoras favorecen el logro de los objetivos de aprendizaje correspondiente al eje de investigación en lengua y literatura. A raíz del análisis de los resultados, se puede señalar que el abordaje del eje de investigación presenta un doble desafío para el profesorado chileno. Por un lado, les demanda fortalecer y profundizar su propia capacidad crítica y reflexiva, y por otro, deberán promoverlo en sus estudiantes.

Lo anterior por tanto, implica al cuerpo docente avanzar desde una perspectiva técnica hacia una más praxiológica, es decir, no basta con otorgar espacios para la indagación, sino que dichos espacios requieren de una intencionalidad pedagógica clara, puesto que deben potenciar el pensamiento crítico en sus estudiantes, para así, formar personas responsables, informadas, comprometidas socialmente y con opiniones sustentadas en argumentos sólidos, donde la investigación adopta un rol fundamental no sólo para la asignatura de Lengua y Literatura, sino que también para las otras asignaturas del plan de estudio, al potenciar habilidades como la comprensión, síntesis, inferencias, análisis y la comparación. En otras palabras, los profesores y profesoras podrán desarrollar el eje de investigación en lengua y literatura, en la medida que ellos mismos investiguen y reflexionen sobre su quehacer pedagógico y cambien sus prácticas en función del grupo de estudiantes, los propósitos que buscan alcanzar y el rol que otorguen al alumnado en la construcción de su propio aprendizaje.

El desafío para el profesorado estaría centrado en planificar actividades que realmente potencien la investigación, en donde utilicen variados tipos de textos y soportes, y no sólo 
hagan usos de textos no literarios. También es importante recalcar que este eje al ser de reciente incorporación en el currículo chileno y no estar considerado en las evaluaciones estandarizadas, tiende a relegarse a un segundo plano, puesto que se privilegia el eje de lectura que sí es considerado.

En palabras simples, potenciar las habilidades de investigación, implica mucho más que la voluntad del cuerpo docente por querer hacerlo, implica conocer a cabalidad el currículo, tener una mirada sistémica de la educación y por supuesto, generar cambios profundos en la formación inicial docente, para que desde los primeros años de docencia, los futuros formadores conozcan la importancia de promover y desarrollar procesos investigativos en el aula y sepan también como potenciar la investigación en y desde la disciplina. También conllevaría a realizar una revisión y actualización de los Estándares orientadores para las carreras de pedagogía de educación media, elaborados por el Ministerio de Educación (2012), puesto que, tanto en los estándares pedagógicos, comunes a todas las pedagogías, como en los estándares disciplinarios de lenguaje y comunicación; es escasa la alusión que se hace a la investigación de manera general, y prácticamente nula a la investigación dentro de la disciplina.

Finalmente, se puede señalar que el estudio presenta algunas limitaciones, entre ellas, el hecho de abordar las prácticas pedagógicas declaradas, es decir, conocer cómo abordan el eje de investigación en lengua y literatura desde el discurso y no desde la acción en aula. Pese a lo anterior, la investigación presenta información relevante acerca de cómo el personal docente de Lengua y Literatura en Chillán-Chile, está abordando el eje de investigación en su disciplina, esto, principalmente por el escaso número de investigaciones que abordan la temática y por la evidente necesidad de potenciar y desarrollar las habilidades de investigación y pensamiento crítico. En consecuencia, algunas líneas de continuidad de esta investigación podrían ser: contrastar el discurso del cuerpo docente con el discurso del estudiantado, analizar cómo se aborda la investigación en la disciplina en la formación inicial docente, de manera de conocer como las diferentes casas de estudios superior, presentan y declaran en su plan de estudio, perfiles de egreso y propósitos de las carreras, todo lo relacionado con las habilidades de investigación en el área disciplinar. Por último, elaborar una propuesta didáctica de investigación en la disciplina, con la participación de diferentes agentes, como estudiantado de pedagogía, profesorado de establecimientos educacionales y personas especialistas en el área de la investigación y la didáctica de la Lengua y la Literatura. 


\section{Agradecimientos}

A los establecimientos educacionales de la ciudad de Chillán, al equipo directivo, y en especial al profesorado de Lengua y Literatura por participar de la investigación.

\section{Referencias}

Ayala-Carabajo, Raquel. (2018). La relación pedagógica: en las fuentes de la experiencia educativa con van Manen. Revista Complutense de Educación, 29(1), 27-41. doi: http://dx.doi.org/10.5209/RCED.51925

Beghadid, Halima. (2013). El enfoque comunicativo, una mejor guía para la práctica docente. Recuperado $\quad$ de https://cvc.cervantes.es/ensenanza/biblioteca ele/publicaciones centros/PDF/oran 20 $13 / 16$ beghadid.pdf

Buendía-Arias, Ximena Paola., Zambrano-Castillo, Lilian Cecilia., y Alirio, Edgar. (2018). El desarrollo de competencias investigativas de los docentes en formación en el contexto de la práctica pedagógica. Folios, (47), 179-195. Recuperado de http://www.scielo.org.co/scielo.php?script=sci arttext\&pid=S0123$48702018000100179 \& \operatorname{lng}=e n \& \operatorname{lng}=e s$.

Camps, Anna. (2012). La investigación en didáctica de la lengua en la encrucijada de muchos caminos. Revista Interamericana de Educación, (59). doi: https://doi.org/10.35362/rie590455

Casanova, Roberto., y Roldán, Yasna. (2016). Alcances sobre la didáctica de la expresión oral y escrita en el aula de enseñanza media. Estudios pedagógicos, 42(Número especial), 41-55. Recuperado de https://scielo.conicyt.cl/scielo.php?script=sci abstract\&pid=S0718$\underline{07052016000300005 \& \operatorname{lng}=e s \& n r m=i s o}$

Chrobak, Ricardo. (2017). El aprendizaje significativo para fomentar el pensamiento crítico. Archivos de Ciencias de la Educación, 11(12), e031. Recuperado de http://www.memoria.fahce.unlp.edu.ar/art revistas/pr.8292/pr.8292.pdf

Colomer, Teresa. (1991). De la enseñanza de la literatura a la educación literaria. Comunicación, Lenguaje y Educación, 9, 1-31. Recuperado de https://dialnet.unirioja.es/servlet/articulo?codigo $=126236$

Colomer, Teresa. (2010). La didáctica de la literatura: temas y líneas de investigación e innovación. Alicante, España: Biblioteca Virtual Miguel de Cervantes. Recuperado de https://www.biblioteca.org.ar/libros/155187.pdf

Díaz, Claudio., Solar, María., Soto, Valentina., Conejeros, Marianela. y Vergara, Jorge. (2015). Temas clave en la formación de profesores en Chile desde la perspectiva de docentes y directivos. Revista Complutense de Educación, 26(3), 543-569. doi: http://dx.doi.org/10.5209/rev RCED.2015.v26.n3.44300 
Dooley, Caitlin., y Assaf, Lori. (2009). Contexts Matter: Two Teachers' Language Arts Instruction in this High-stakes Era. Journal of Literacy Research, 41(3), 354-391.doi: https://doi.org/10.1080\%2F10862960903133743

Escobar-Pérez, Jazmine., y Cuervo-Martínez, Ángela. (2008). Validez de contenido y juicio de expertos: una aproximación a su utilización. Avances en Medición, 6, 27-36. Recuperado de

http://www.humanas.unal.edu.co/psicometria/files/7113/8574/5708/Articulo3 Juicio de expertos 27-36.pdf

Espinosa, María Jesús., y Concha, Soledad. (2015). Aprendizaje de la escritura en las nuevas bases curriculares de Lenguaje y Comunicación: Nociones teóricas y modelos de escritura que subyacen a la propuesta curricular. Estudios pedagógicos, 41(2), 325-344. doi: https://doi.org/10.4067/S0718-07052015000200019

Falabella, Alejandra., y Opazo, Catalina. (2014). Sistema de Aseguramiento de la Calidad y procesos de mejora: Una mirada desde la gestión educativa: Informe ejecutivo. Santiago de Chile: CIDE; Universidad Alberto Hurtado. Recuperado de https://bit.ly/3kxaUvc

Flick, Uwe. (2012). Introducción a la investigación cualitativa. Madrid: Morata.

Giraldo, Camilo. (2015). La escritura en el aula como instrumento de aprendizaje. Estudio en universidades. Ánfora, 22(38), 39-60. Recuperado de https://bit.ly/3jvIVL6

Hernández, Roberto., Fernández, Carlos., y Baptista, María del Pilar. (2014). Metodología de la investigación (6 ${ }^{\mathrm{a}}$ ed.). Distrito Federal, México: McGraw-Hill.

Iturra, Carolina. (2015). La organización de la enseñanza de la lectura en las aulas chilenas. Diferencias entre profesores calificados como básicos o como competentes. Universitas Psychologica, 14(2), 535-548. doi: https://doi.org/10.11144/Javeriana.upsy14-2.oela

Manzi, Jorge., Bogolasky, Francisca., Gutiérrez, Gabriel., Grau, Valeska., y Volante Paulo. (2014). Análisis sobre valoraciones, comprensión y uso del SIMCE por parte de directores escolares de establecimientos subvencionados. Chile: Fondo de Investigación y Desarrollo en Educación. Recuperado de https://politicaspublicas.uc.cl/wpcontent//uploads/2016/04/Informe-Final-F711269-Manzi.pdf

Martínez, Dianelkys., y Marquéz, Dora. (2015). Las habilidades investigativas como eje transversal de la formación para la investigación. Tendencias pedagógicas, 24, 347 360. Recuperado de https://revistas.uam.es/tendenciaspedagogicas/article/view/2110

Mendoza, Antonio (Ed.). (2003). Didáctica de la Lengua y la Literatura. Madrid, España: Prentice Hall.

Milián, Marta. (2013). La enseñanza de la composición escrita. En Didáctica de la lengua castellana y la literatura (pp. 121- 138). Madrid, España: Ministerio de Educación de España; Editorial GRAÓ. 
Ministerio de Educación del Gobierno de Chile. (2012). Estándares Orientadores para Carreras de Pedagogía de Educación Media. Santiago, Chile: Ministerio de Educación. Recuperado de https://www.cpeip.cl/wpcontent/uploads/2019/03/Est\%C3\%A1ndares Media.pdf

Ministerio de Educación del Gobierno de Chile. (2015). Bases curriculares $7^{\circ}$ a $2^{\circ}$ medio. Recuperado de https://www.curriculumnacional.cl/614/articles-37136 bases.pdf

Ministerio de Educación del Gobierno de Chile. (2016). Lengua y Literatura Programa de Estudio Primero Medio. Recuperado de https://www.curriculumnacional.cl/portal/Educacion-General/Lenguaje-y-comunicacionLengua-y-literatura/Lengua-y-literatura-1-Medio/

Moreno, María. (2005). Potenciar la educación. un currículum transversal de formación para la investigación. REICE. Revista Iberoamericana sobre Calidad, Eficacia y Cambio en Educación, 3(1), 520-540. Recuperado de https://www.redalyc.org/pdf/551/55130152.pdf

Munita, Felipe., y Margallo, Ana. (2019). La didáctica de la literatura. Perfiles Educativos, 41(164), 154-170. doi: https://doi.org/10.22201/iisue.24486167e.2019.164.58825

Muñoz, José., Quintero, Josefina., y Munévar, Raúl. (2001). Cómo desarrollar competencias investigativas en educación. Bogotá, Colombia: Cooperativa Editorial Magisterio.

Organización para la Cooperación y el Desarrollo Económicos (OCDE). (2015). Panorama de La Educación 2014: Indicadores de La OCDE. España: OCDE; Fundación Santillana. doi: https://doi.org/10.1787/eag-2014-es

Paul, Richard., y Elder, Linda. (2005). Estándares de competencia para el pensamiento crítico. Estándares, Principios, Desempeño, Indicadores y Resultados con una Rúbrica Maestra en el Pensamiento Crítico. Fundación para el Pensamiento Crítico. Recuperado de http://eduteka.icesi.edu.co/pdfdir/EstandaresPensamientoCritico ESPANHOL.pdf

Pérez, Coralia., y López, Lutgarda. (1999). Las habilidades e invariantes investigativas en la formación del profesorado. Una propuesta metodológica para su estudio. La Habana, Cuba: Editorial Universitaria.

Quecedo, Rosario., y Castaño, Carlos. (2002). Introducción a la metodología de investigación cualitativa. Revista de Psicodidáctica, (14), 5-39. Recuperado de https://www.redalyc.org/articulo.oa?id=17501402

Robles, Pilar., y Rojas, Manuela del Carmen. (2015). La validación por juicio de expertos: dos investigaciones cualitativas en Lingüística aplicada. Revista Nebrija de Lingüística Aplicada a la Enseñanza de las Lenguas, (18). Recuperado de https://www.nebrija.com/revistalinguistica/files/articulosPDF/articulo 55002aca89c37.pdf

Rojas, Elvis., Naranjo, Josué., Campos, Raúl. y Campos, Gloria. (2018). Enseñanza de la programación: la importancia de promover actitudes autodidactas en los estudiantes. Atenas, 4(44), 46-59. Recuperado de https://www.redalyc.org/jatsRepo/4780/478055154003/html/index.html 
Ruiz, Jorge. (2010). Importancia de la investigación. Revista Científica, XX(2), 125-126. Recuperado de https://www.redalyc.org/pdf/959/95912322001.pdf

Ruiz, José. (2012). Metodología de la investigación cualitativa (5a ed. ). Bilbao, España: Universidad de Deusto.

Ruiz, Uri (Coord.). (2013). El objeto de la didáctica de la lengua y la literatura. Didáctica de la lengua castellana y la literatura (pp. 11-33). Madrid, España: Ministerio de Educación de España; Editorial GRAÓ.

Sánchez, Manuel., y del Sagrario Corte, Francisca. (2013). Las evaluaciones estandarizadas: sus efectos en tres países latinoamericanos. Revista Latinoamericana de Estudios Educativos (México), XLIII(1), 97-124. Recuperado de https://www.redalyc.org/articulo.oa?id=270/27026416001

Silva, Macarena. (2014). El estudio de la comprensión lectora en Latinoamérica: necesidad de un enfoque en la comprensión. Innovación Educativa, 14(64), 47-56. Recuperado de http://www.scielo.org.mx/pdf/ie/v14n64/v14n64a5.pdf

Silva, Miroslava., García, Teresa., Guzmán, Teresa., Chaparro, Ricardo. (2016). Estudio de herramientas Moodle para desarrollar habilidades del siglo XXI. Campus Virtuales, 5(2), 58-69. Recuperado de http://uajournals.com/ojs/index.php/campusvirtuales/article/view/126/131

Soto, María. (2018). La formación de habilidades investigativas en los futuros profesores de educación inicial. Revista Senderos Pedagógicos, 8(8), 111-121. Recuperado de https://ojs.tdea.edu.co/index.php/senderos/article/view/532

Torres, R. (1999). El maestro investigador, la investigación en el aula. Barcelona, España: Grao.

Van Manen, Max. (2003). Investigación educativa y experiencia vivida. Ciencia humana para una pedagogía de la acción y sensibilidad. Barcelona, España: Idea Books.

Vilà, Montserrat. (2005). El discurso oral formal contenidos de aprendizaje y secuencias didácticas. Barcelona, España: Graó

Viñao, Antonio. (2018). La desprofesionalización de la docencia: viejas cuestiones, nuevas amenazas. Revista digital de educación del FEAE-Aragón, VIII(23), 8 - 12. Recuperado de http://feae.eu/wp-content/uploads/2018/03/Forum-Arag\%C3\%B3n-23.pdf

Vital, Marisela. (2015). La investigación en los procesos de enseñanza aprendizaje. Vida Científica Boletín Científico De La Escuela Preparatoria No. 4, 3(6). Recuperado de https://repository.uaeh.edu.mx/revistas/index.php/prepa4/article/view/1980

Zebadúa, María de Lourdes, y García, Ernesto. (2012). Cómo enseñar a hablar y escuchar en el salón de clases. México: Universidad Nacional Autónoma de México. Recuperado de https://portalacademico.cch.unam.mx/materiales/libros/pdfs/librocch hablarescuchar.pd $\underline{f}$ 
Revista indizada en

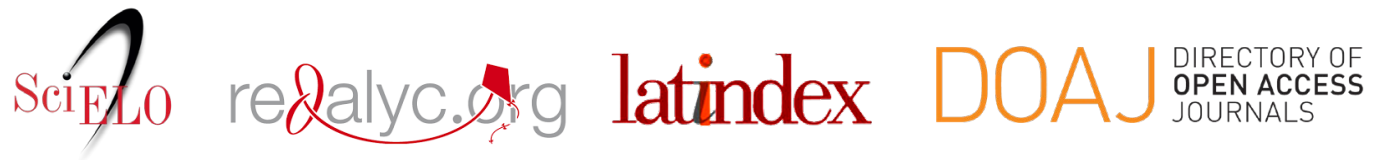

Distribuida en las bases de datos:

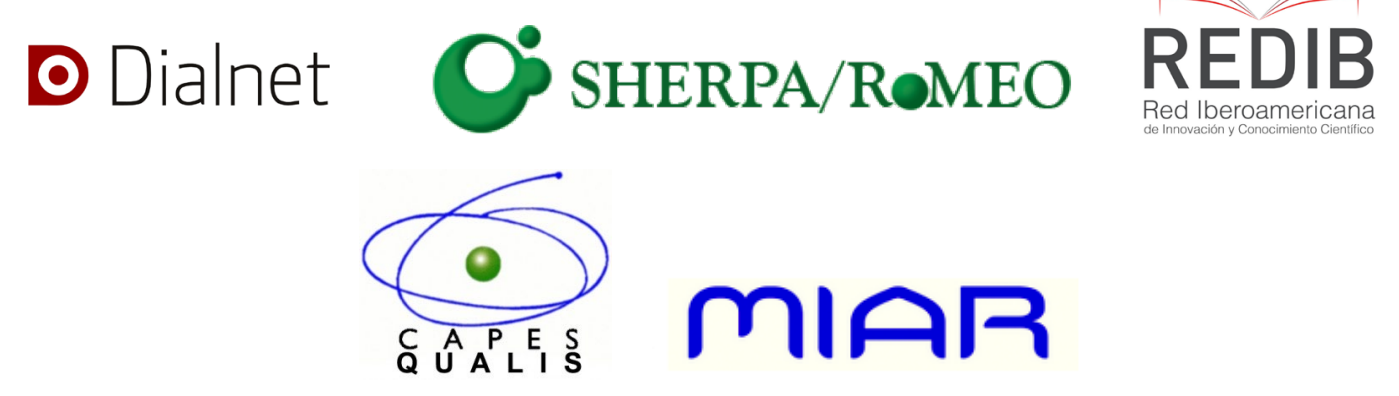

